\title{
HOMOGENIZATION OF A DARCY-STOKES SYSTEM MODELING VUGGY POROUS MEDIA
}

\author{
TODD ARBOGAST* AND HEATHER L. LEHR ${ }^{\dagger}$
}

\begin{abstract}
We derive a macroscopic model for single phase, incompressible, viscous fluid flow in a porous medium with small cavities called vugs. We model the vuggy medium on the microscopic scale using Stokes equations within the vugular inclusions, Darcy's law within the porous rock, and a Beavers-Joseph-Saffman boundary condition on the interface between the two regions. We assume periodicity of the medium, and obtain uniform energy estimates independent of the period. Through a two-scale homogenization limit as the period tends to zero, we obtain a macroscopic Darcy's law governing the medium on larger scales. We also develop some needed generalizations of the two-scale convergence theory needed for our bi-modal medium, including a two-scale convergence result on the Darcy-Stokes interface. The macroscopic Darcy permeability is computable from the solution of a cell problem. An analytic solution to this problem in a simple geometry suggests that: (1) flow along vug channels is primarily Poiseuille with a small perturbation related to the Beavers-Joseph slip, and (2) flow that alternates from vug to matrix behaves as if the vugs have infinite permeability.
\end{abstract}

Key words. Homogenization, two-scale convergence, Darcy-Stokes system, vuggy porous media, Beavers-Joseph boundary condition

1. Introduction. A vug is a cavity in a porous medium that is relatively larger than the intergranular pore space. Vugular inclusions are especially common in carbonate rocks, and are endemic to many of the world's groundwater aquifers and petroleum reservoirs. Although small, vugs can significantly increase both the effective porosity and permeability of the medium. We consider in this paper a porous medium with many small vugs scattered throughout its extent.

It is well established, both empirically and theoretically, that Darcy's law governs fluid flow in a porous medium on scales above the pore diameter [6, 23, 27, 29]. Since the flow is expected to have a relatively low Reynolds number, the Stokes equations should adequately model fluid flow in the vugs.

In 1967, Beavers and Joseph [7] determined experimentally that a free fluid in contact with a porous medium flows faster than a fluid in contact with a completely solid surface. Although thin boundary layers arise in both cases, the latter case is generally modeled by assuming that all components of the velocity vanish at the solid contact surface. In the former case, the experiments of Beavers and Joseph demonstrate that the tangential velocity of the fluid cannot vanish. They proposed to account for this slippage by imposing a boundary condition of the form

$$
\frac{\partial U_{s}}{\partial y}=\frac{\alpha}{\sqrt{K}}\left(U_{s}-U_{d}\right)
$$

where $\partial / \partial y$ is the normal derivative, $U_{s}$ is the tangential component of the Stokes velocity, $U_{d}$ is the tangential component of the Darcy velocity, $K$ is the permeability of the porous medium, and $\alpha$ is the dimensionless Beavers-Joseph slippage coefficient. Saffman [24] justified this law theoretically, and showed that the term involving $U_{d}$ could be dropped (see also $[15,16])$. Jones [18] reinterpreted this law so that it applies

\footnotetext{
*Department of Mathematics, C1200; and Institute for Computational Engineering and Sciences, C0200; The University of Texas at Austin; Austin, TX 78712 (arbogast@ices.utexas.edu).

${ }^{\dagger}$ Department of Mathematics, C1200; The University of Texas at Austin; Austin, TX 78712; current address: Department of Mathematics, The Ohio State University, 231 W. 18th Avenue, Columbus, OH 43210 (heather@math.ohio-state.edu).
} 
to curved boundaries and nontangential flows by formulating the boundary condition in terms of the tangential component of the fluid stress tensor (see (1.6) below).

We model the vuggy medium on the fine scale using Stokes equations in the vugs, Darcy's law in the porous rock, and the Beavers-Joseph-Saffman boundary condition on the interface between the two. We assume periodicity of the medium, and obtain as our homogenized limit a macroscopic Darcy's law governing the system over large scales. To illustrate the ideas, we first derive this macroscopic model formally in $\S 2$, and then we derive it rigorously by the two-scale convergence method $[22,4,1,14]$ in $\S 5$. In $\S 3$ we obtain the existence, uniqueness, and energy estimates results needed in the analysis. In $\S 4$ we develop the needed generalizations of the two-scale convergence theory needed for our bi-modal medium. The convergence of the homogenization is demonstrated in $\S 5$. The final section presents a simple analytical solution to illustrate the results.

Although our results would extend easily to $\mathbb{R}^{3}$, for ease of presentation we assume that the domain $\Omega$ is Lipschitz and bounded in $\mathbb{R}^{2}$. We assume that the geometric vug and pore structure of $\Omega$ is periodic of period $\epsilon Y$, where $Y$ is a reference cell for the periodic tiling of unit volume $|Y|$. The portion of the domain consisting of the vugs is denoted $\Omega_{s}^{\epsilon}$, and that consisting of the porous rock is $\Omega_{d}^{\epsilon}$. Let $\Gamma^{\epsilon}$ be the interface between the two regions. Let $\eta_{s}$ be the outer unit normal to $\partial \Omega_{s}$, and let $\tau$ be a unit tangent to $\Gamma^{\epsilon}$.

Let $D$ be the symmetric gradient, i.e., $D(\psi)$ is the matrix $\frac{1}{2}\left(\frac{\partial \psi_{i}}{\partial x_{j}}+\frac{\partial \psi_{j}}{\partial x_{i}}\right)$. Denote by $\mu>0$ the fluid viscosity; $K^{\epsilon}=K(x / \epsilon)$ the $Y$-periodic, bounded, symmetric and uniformly positive definite permeability tensor of the porous rock matrix; and $\alpha>0$ the Beavers-Joseph slip coefficient. The fluid velocity and pressure in the Stokes and Darcy regions are denoted $u_{s}^{\epsilon}, p_{s}^{\epsilon}$ and $u_{d}^{\epsilon}, p_{d}^{\epsilon}$, respectively. These satisfy the following set of equations (wherein $q \in L^{2}(\Omega)$ is an external source or sink satisfying the compatibility condition that its average over $\Omega$ vanishes, and $f \in\left(L^{2}(\Omega)\right)^{2}$ is a term related to body forces such as gravitation):

Vugular region (Stokes equations)

$$
\begin{aligned}
-2 \mu \epsilon^{2} \nabla \cdot D u_{s}^{\epsilon}+\nabla p_{s}^{\epsilon} & =f & & \text { in } \Omega_{s}^{\epsilon}, \\
\nabla \cdot u_{s}^{\epsilon} & =q & & \text { in } \Omega_{s}^{\epsilon},
\end{aligned}
$$

Rock matrix (Darcy equations)

$$
\begin{array}{rlrl}
\mu\left(K^{\epsilon}\right)^{-1} u_{d}^{\epsilon}+\nabla p_{d}^{\epsilon} & =f & & \text { in } \Omega_{d}^{\epsilon}, \\
\nabla \cdot u_{d}^{\epsilon}=q & & \text { in } \Omega_{d}^{\epsilon}
\end{array}
$$

Interface

$$
\begin{aligned}
u_{s}^{\epsilon} \cdot \eta_{s} & =u_{d}^{\epsilon} \cdot \eta_{s} & & \text { on } \Gamma^{\epsilon}, \\
2 \eta_{s} \cdot D u_{s}^{\epsilon} \cdot \tau & =-\frac{\alpha}{\epsilon \sqrt{K^{\epsilon}}} u_{s}^{\epsilon} \cdot \tau & & \text { on } \Gamma^{\epsilon}, \\
2 \mu \epsilon^{2} \eta_{s} \cdot D u_{s}^{\epsilon} \cdot \eta_{s} & =p_{s}^{\epsilon}-p_{d}^{\epsilon} & & \text { on } \Gamma^{\epsilon},
\end{aligned}
$$

Outer boundary

$$
\begin{aligned}
u_{s}^{\epsilon}=0 & \text { on } \partial \Omega \cap \partial \Omega_{s}^{\epsilon}, \\
u_{d}^{\epsilon} \cdot \eta=0 & \text { on } \partial \Omega \cap \partial \Omega_{d}^{\epsilon} .
\end{aligned}
$$


The interface conditions represent continuity of mass flux (1.5), the Beavers-JosephSaffman condition on the tangential stress (1.6), and the continuity of normal stress (1.7). Examples of direct numerical simulation of these equations in a non-vuggy context (i.e., in a medium with at most a few very large vugs) can be found in, e.g., $[25,13,19]$. In a vuggy context, see, e.g., [3].

The homogenization problem is to determine the behavior of the system as $\epsilon \rightarrow 0$. Note that in the equations we have scaled both the viscosity $\mu$ and the permeability $K^{\epsilon}$ by $\epsilon^{2}$. This is the usual scaling for deriving Darcy's law from Stokes flow (see [27]), since as $\epsilon \rightarrow 0$, flow paths (in our case vugs) become constricted, so a corresponding decrease in viscosity is required to maintain flow rates. Moreover, when homogenizing heterogeneity (see, e.g., $[8,26]$ ), the ratio of permeability to viscosity should be fixed, forcing a similar scaling of the permeability. These considerations then imply the stated scaling of the Beavers-Joseph boundary condition.

Below we will need to distinguish the geometry of the reference cell $Y$, so let $Y_{s}$ denote the Stokes region, $Y_{d}$ the Darcy region, and $\Gamma$ the interface between the two. We assume that both $Y_{s}$ and $Y_{d}$ have positive measure, and thus also the 1-dimensional measure of $\Gamma$ is positive. As usual, $x$ will represent a point in $\Omega$ and $y$ a point in $Y$. In the sequel, let $\|\cdot\|_{\omega}$ and $(\cdot, \cdot)_{\omega}$ denote the $L^{2}(\omega)$ norm and inner-product, respectively, where we omit the domain $\omega$ if it is $\Omega$.

2. Formal Homogenization. We proceed to formally homogenize our system of equations in the usual manner $[8,14,17,26]$. We make the ansatz that we can expand $u_{\ell}^{\epsilon}$ and $p_{\ell}^{\epsilon}($ for $\ell=s, d)$ as

$$
u_{\ell}^{\epsilon}=\sum_{j=0}^{\infty} \epsilon^{j} u_{\ell, j}\left(x, \frac{x}{\epsilon}\right) \quad \text { and } \quad p_{\ell}^{\epsilon}=\sum_{j=0}^{\infty} \epsilon^{j} p_{\ell, j}\left(x, \frac{x}{\epsilon}\right)
$$

where the $u_{\ell, j}(x, y)$ and $p_{\ell, j}(x, y)$ are $Y$-periodic functions in $y$.

Substituting the above expressions into our system of equations (1.1)-(1.7), and recognizing that $\nabla=\nabla_{x}+\epsilon^{-1} \nabla_{y}$, we obtain the following equations. From the $\epsilon^{-1}$ terms of (1.1) and (1.3), and the $\epsilon^{0}$ terms of (1.7), we see that

$$
\begin{aligned}
\nabla_{y} p_{s}^{0}=0 & \text { in } \Omega \times Y_{s}, \\
\nabla_{y} p_{d}^{0}=0 & \text { in } \Omega \times Y_{d}, \\
p_{s}^{0}-p_{d}^{0}=0 & \text { on } \Omega \times \Gamma .
\end{aligned}
$$

It follows immediately that $p_{s}^{0}$ and $p_{d}^{0}$ are independent of $y$ and equal, so let

$$
p^{0}(x)=p_{s}^{0}(x)=p_{d}^{0}(x) \quad \text { on } \Omega
$$

Now the $\epsilon^{0}$ terms of (1.1), (1.3) and (1.5), the $\epsilon^{-1}$ terms of (1.2), (1.4) and (1.6), 
and the $\epsilon^{1}$ terms of (1.7) imply

$$
\begin{aligned}
& -2 \mu \nabla_{y} \cdot D_{y} u_{s}^{0}+\nabla_{x} p^{0}(x)+\nabla_{y} p_{s}^{1}(x, y)=f \quad \text { in } \Omega \times Y_{s}, \\
& \nabla_{y} \cdot u_{s}^{0}=0 \quad \text { in } \Omega \times Y_{s}, \\
& \mu K(y)^{-1} u_{d}^{0}+\nabla_{x} p^{0}(x)+\nabla_{y} p_{d}^{1}(x, y)=f \quad \text { in } \Omega \times Y_{d}, \\
& \nabla_{y} \cdot u_{d}^{0}=0 \quad \text { in } \Omega \times Y_{d}, \\
& u_{s}^{0} \cdot \eta_{s}=u_{d}^{0} \cdot \eta_{s} \quad \text { on } \Omega \times \Gamma, \\
& 2 \eta_{s} \cdot D_{y} u_{s}^{0} \cdot \tau=-\frac{\alpha}{\sqrt{K(y)}} u_{s}^{0} \cdot \tau \quad \text { on } \Omega \times \Gamma, \\
& 2 \mu \eta_{s} \cdot D_{y} u_{s}^{0} \cdot \eta_{s}=p_{s}^{1}-p_{d}^{1} \quad \text { on } \Omega \times \Gamma .
\end{aligned}
$$

With $e_{j}$ being the standard Cartesian basis vector in the $j$ th direction, let $\left(\omega_{j}, \Phi_{j}\right)$ be the periodic solution of the following auxiliary or cell problem

$$
\begin{aligned}
-2 \nabla \cdot D \omega_{j}^{s}+\nabla \Phi_{j}^{s} & =e_{j} & & \text { in } Y_{s}, \\
\nabla \cdot \omega_{j}^{s} & =0 & & \text { in } Y_{s}, \\
K^{-1} \omega_{j}^{d}+\nabla \Phi_{j}^{d} & =e_{j} & & \text { in } Y_{d}, \\
\nabla \cdot \omega_{j}^{d} & =0 & & \text { in } Y_{d}, \\
\omega_{j}^{s} \cdot \eta_{s} & =\omega_{j}^{d} \cdot \eta_{s} & & \text { on } \Gamma, \\
2 \eta_{s} \cdot D \omega_{j}^{s} \cdot \tau & =-\frac{\alpha}{\sqrt{K}} \omega_{j}^{s} \cdot \tau & & \text { on } \Gamma, \\
2 \eta_{s} \cdot D \omega_{j}^{s} \cdot \eta_{s} & =\Phi_{j}^{s}-\Phi_{j}^{d} & & \text { on } \Gamma .
\end{aligned}
$$

Then by linear algebra, we can express $u_{s}^{0}$ and $u_{d}^{0}$ as

$$
u_{\ell}^{0}(x, y)=\frac{1}{\mu} \sum_{j}\left(f_{j}(x)-\frac{\partial p^{0}}{\partial x_{j}}(x)\right) \omega_{j}^{\ell}(y), \ell=s, d .
$$

Define the averaging operator $\bar{v}_{\ell}$ by averaging $v_{\ell}$ in the following sense

$$
\bar{v}_{\ell}=\frac{1}{|Y|} \int_{Y_{\ell}} v_{\ell}(y) d y
$$

so that

$$
\bar{u}_{0}(x)=\bar{u}_{s}^{0}(x)+\bar{u}_{d}^{0}(x)=\frac{1}{\mu} \sum_{j}\left(f_{j}(x)-\partial_{j} p^{0}(x)\right)\left(\bar{\omega}_{j}^{s}+\bar{\omega}_{j}^{d}\right) .
$$

Now let the matrix $\tilde{K}$ be defined by

$$
\tilde{K}_{i, j}=\bar{\omega}_{j, i}^{s}+\bar{\omega}_{j, i}^{d}=\frac{1}{|Y|}\left(\int_{Y_{d}}\left(\omega_{j}^{d}\right)_{i} d y+\int_{Y_{s}}\left(\omega_{j}^{s}\right)_{i} d y\right) .
$$

Then we see that

$$
\mu \tilde{K}^{-1} \bar{u}_{0}+\nabla p^{0}=f \quad \text { in } \Omega .
$$


Finally, using the $\epsilon^{0}$ terms of (1.2) and (1.4), we obtain

$$
\nabla_{x} \cdot u_{\ell}^{0}+\nabla_{y} \cdot u_{\ell}^{1}=q \quad \text { in } \Omega \times Y_{s}, \ell=s, d,
$$

so that if we again average over $Y$ and sum, we obtain

$$
\begin{aligned}
\nabla \cdot \bar{u}_{0} & +\frac{1}{|Y|} \int_{Y_{s}} \nabla_{y} \cdot u_{s}^{1} d y+\frac{1}{|Y|} \int_{Y_{d}} \nabla_{y} \cdot u_{d}^{1} d y \\
= & \nabla \cdot \bar{u}_{0}+\frac{1}{|Y|} \int_{\partial Y_{s}} u_{s}^{1} \cdot \eta_{s} d S+\frac{1}{|Y|} \int_{\partial Y_{d}} u_{d}^{1} \cdot \eta_{d} d S \\
& =q .
\end{aligned}
$$

By the periodicity of $u_{\ell}^{1}$ in $y$, and the fact that $u_{s}^{1} \cdot \eta_{s}=-u_{d}^{1} \cdot \eta_{d}$ on $\Gamma$, we see that

$$
\nabla \cdot \bar{u}_{0}=q \quad \text { on } \Omega .
$$

Thus we conclude from the formal analysis that $\bar{u}_{0}$ should satisfy a Darcy's law on all of $\Omega(2.14)-(2.15)$, with effective permeability matrix $\tilde{K}$, independent of the fluid viscosity and given by (2.13).

Lemma 2.1. The tensor $\tilde{K}$, as defined by (2.13) and (2.4)-(2.10), is symmetric and positive definite.

Proof. The existence and uniqueness of a weak solution to (2.4)-(2.10) follows from an analysis similar to that given below for Theorem 3.1. Note that (2.4)-(2.10) is equivalent to the variational equation

$$
2\left(D \omega_{i}^{s}, D \psi\right)_{Y_{s}}+\left(\frac{\alpha}{\sqrt{K}} \omega_{i}^{s} \cdot \tau, \psi \cdot \tau\right)_{\Gamma}+\left(K^{-1} \omega_{i}^{d}, \psi\right)_{Y_{d}}=\left(e_{i}, \psi\right)_{Y},
$$

for $\psi$ an infinitely differentiable and periodic vector function in $Y$ such that $\nabla \cdot \psi=0$. With $\psi=\omega_{j}^{s}$ on $Y_{s}$ and $\psi=\omega_{j}^{d}$ on $Y_{d}$ (actually a sequence approaching the same), we obtain that

$$
\begin{aligned}
|Y| \tilde{K}_{i, j} & =\left(e_{i}, \omega_{j}^{s}\right)_{Y_{s}}+\left(e_{i}, \omega_{j}^{d}\right)_{Y_{d}} \\
& =2\left(D \omega_{i}^{s}, D \omega_{j}^{s}\right)_{Y_{s}}+\left(\frac{\alpha}{\sqrt{K}} \omega_{i}^{s} \cdot \tau, \omega_{j}^{s} \cdot \tau\right)_{\Gamma}+\left(K^{-1} \omega_{i}^{d}, \omega_{j}^{d}\right)_{Y_{d}},
\end{aligned}
$$

and symmetry follows immediately.

To show that $\tilde{K}$ is positive definite, take any $\lambda \in \mathbb{R}^{2}$ and define

$$
\xi^{\ell}(y)=\sum_{i} \lambda_{i} \omega_{i}^{\ell} \quad \text { for } y \in Y_{\ell} .
$$

Then, from (2.16), we conclude that

$$
|Y| \lambda^{T} \tilde{K} \lambda=2\left(D \xi^{s}, D \xi^{s}\right)_{Y_{s}}+\left(\frac{\alpha}{\sqrt{K}} \xi^{s} \cdot \tau, \xi^{s} \cdot \tau\right)_{\Gamma}+\left(K^{-1} \xi^{d}, \xi^{d}\right)_{Y_{d}},
$$

and that $\tilde{K}$ is positive semi-definite. To see definiteness, suppose that $\lambda^{T} \tilde{K} \lambda=0$ and conclude that each integrand above vanishes. But now (2.16) implies that

$$
0=2\left(D \xi^{s}, D \psi\right)_{Y_{s}}+\left(\frac{\alpha}{\sqrt{K}} \xi^{s} \cdot \tau, \psi \cdot \tau\right)_{\Gamma}+\left(K^{-1} \xi^{d}, \psi\right)_{Y_{d}}=(\lambda, \psi)_{Y} .
$$

Since $\lambda$ is constant, we can take $\psi=\lambda$ and conclude that $\lambda=0$ and, further, that $\tilde{K}$ is positive definite. 
3. Existence and A Priori Energy Estimates. In this section, we prove a theorem which gives existence of solutions $u^{\epsilon}$ and $p^{\epsilon}$ to (1.1)-(1.9) for each $\epsilon$ and energy estimates for $u^{\epsilon}$ and $p^{\epsilon}$ independent of $\epsilon$. Let

$$
\begin{aligned}
& V^{\epsilon}=V^{\epsilon}(\Omega)=\left\{v \in H(\operatorname{div}, \Omega)\left|v_{s}=v\right|_{\Omega_{s}^{\epsilon}} \in H^{1}\left(\Omega_{s}^{\epsilon}\right)\right\}, \\
& V_{0}^{\epsilon}=V_{0}^{\epsilon}(\Omega)=\left\{v \in V^{\epsilon}(\Omega) \mid v \cdot \eta=0 \text { on } \partial \Omega \cap \partial \Omega_{d}^{\epsilon} \text { and } v=0 \text { on } \partial \Omega \cap \partial \Omega_{s}^{\epsilon}\right\},
\end{aligned}
$$

where $\eta$ is the outward unit normal to $\Omega$, and let $W=L^{2}(\Omega) / \mathbb{R}$.

We first recast the original problem (1.1)-(1.9) into a variational problem. Combine equations (1.2) and (1.4), multiply by a test function $w \in W$, and integrate over $\Omega$. Then combine and multiply equations (1.1) and (1.3) by a test function $v \in V^{\epsilon}$, integrate, integrate by parts, and manipulate the boundary terms to obtain the variational form of the system for $u^{\epsilon} \in V_{0}^{\epsilon}$ and $p^{\epsilon} \in W$ satisfying

$$
\begin{array}{ll}
2 \mu \epsilon^{2}\left(D u_{s}^{\epsilon}, D v\right)_{\Omega_{s}^{\epsilon}}+\left(\frac{\epsilon \mu \alpha}{\sqrt{K^{\epsilon}}} u_{s}^{\epsilon} \cdot \tau, v_{s} \cdot \tau\right)_{\Gamma^{\epsilon}} & \\
\quad-\left(p^{\epsilon}, \nabla \cdot v\right)_{\Omega}+\mu\left(\left(K^{\epsilon}\right)^{-1} u_{d}^{\epsilon}, v\right)_{\Omega_{d}^{\epsilon}}=(f, v)_{\Omega}, & v \in V_{0}^{\epsilon}, \\
\left(\nabla \cdot u^{\epsilon}, w\right)_{\Omega}=(q, w)_{\Omega}, & w \in W,
\end{array}
$$

where $u_{d}^{\epsilon}=\left.u\right|_{\Omega_{d}^{\epsilon}}$ and $u_{s}^{\epsilon} \cdot \eta_{s}=u_{d}^{\epsilon} \cdot \eta_{s}$ on $\Gamma^{\epsilon}$ is implicit from $u^{\epsilon} \in V_{0}^{\epsilon}$.

ThEOREM 3.1. For each $\epsilon$, there exists $\left(u^{\epsilon}, p^{\epsilon}\right) \in V_{0}^{\epsilon} \times W$ satisfying (1.1)-(1.9) weakly, i.e., (3.1)-(3.2), such that

$$
\epsilon\left\|\nabla u_{s}^{\epsilon}\right\|_{\Omega_{s}^{\epsilon}}+\sqrt{\epsilon}\left\|u_{s}^{\epsilon} \cdot \tau\right\|_{\Gamma^{\epsilon}}+\left\|u^{\epsilon}\right\|+\left\|\nabla \cdot u^{\epsilon}\right\|+\left\|p^{\epsilon}\right\| \leq C(\|f\|+\|q\|),
$$

with $C$ independent of $\epsilon$.

In order to prove this result, we first prove a lemma related to Korn's inequality.

Lemma 3.2. There exists $C$ independent of $\epsilon$ such that for all $v \in V_{0}^{\epsilon}(\Omega)$,

$$
\left\|v_{s}\right\|_{\Omega_{s}^{\epsilon}}+\epsilon\left\|\nabla v_{s}\right\|_{\Omega_{s}^{\epsilon}} \leq C\left(\epsilon\left\|D v_{s}\right\|_{\Omega_{s}^{\epsilon}}+\sqrt{\epsilon}\left\|v_{s} \cdot \tau\right\|_{\Gamma^{\epsilon}}+\left\|v_{d}\right\|_{\Omega_{d}^{\epsilon}}+\epsilon\|\nabla \cdot v\|\right) .
$$

If $\Omega_{d}$ is not empty, the above inequality holds for $v \in V^{\epsilon}(\Omega)$.

Proof. First we show that a similar result holds for $\hat{v} \in V(Y)=\{v \in H(\operatorname{div}, Y) \mid$ $\left.v_{s}=\left.v\right|_{Y_{s}} \in H^{1}\left(Y_{s}\right)\right\}$, and then we use a translation and scaling argument to pass to all of $\Omega$. Suppose it is not true that there exists $\hat{C}$ such that

$$
\left\|\hat{v}_{s}\right\|_{Y_{s}}+\left\|\nabla \hat{v}_{s}\right\|_{Y_{s}} \leq \hat{C}\left(\left\|D \hat{v}_{s}\right\|_{Y_{s}}+\left\|\hat{v}_{s} \cdot \tau\right\|_{\Gamma}+\left\|\hat{v}_{d}\right\|_{Y_{d}}+\|\nabla \cdot \hat{v}\|_{Y}\right) .
$$

Then there exists a sequence $\left\{\hat{v}_{n}\right\}_{n=1}^{\infty} \in V(Y)$ such that

$$
\left\|\hat{v}_{n, s}\right\|_{Y_{s}}+\left\|\nabla \hat{v}_{n, s}\right\|_{Y_{s}}=1
$$

and

$$
\left\|D \hat{v}_{n, s}\right\|_{Y_{s}}+\left\|\hat{v}_{n, s} \cdot \tau\right\|_{\Gamma}+\left\|\hat{v}_{n, d}\right\|_{Y_{d}}+\left\|\nabla \cdot \hat{v}_{n}\right\|_{Y} \leq \frac{1}{n}
$$

The last two terms on the left-hand side above tell us that $\hat{v}_{n, d} \rightarrow 0$ in $H\left(\operatorname{div}, Y_{d}\right)$, and (3.5) implies that $\hat{v}_{n, s} \rightarrow \hat{v}_{s}$ weakly in $H^{1}\left(Y_{s}\right)$ for some $\hat{v}_{s}$. Let $\hat{v}$ be the extension by zero of $\hat{v}_{s}$ to $Y$. Since $\hat{v}_{n}$ is bounded in $H(\operatorname{div}, Y)$, it converges weakly, and we conclude that in fact

$$
\hat{v}_{n} \rightarrow \hat{v} \quad \text { in } H(\operatorname{div}, Y) .
$$


Since

$$
\left\|\hat{v}_{n, d} \cdot \eta\right\|_{\left(H_{00}^{1 / 2}(\Gamma)\right)^{*}} \leq C\left\|\hat{v}_{n, d}\right\|_{H\left(\mathrm{div}, Y_{d}\right)} \rightarrow 0
$$

where the norm on the left-hand side is the norm of the dual space of $H_{00}^{1 / 2}(\Gamma)$ (see [20]), we conclude that $\hat{v}_{d} \cdot \eta=\hat{v}_{s} \cdot \eta=0$ on $\Gamma$. On the other hand, $\left\|\hat{v}_{n, s} \cdot \tau\right\|_{\Gamma} \rightarrow 0$, so that $\hat{v}_{s} \cdot \tau=0$ and further that $\hat{v}_{s}=0$ on $\Gamma$. Now Korn's inequality can be applied on $Y_{s}$ to show that

$$
\left\|\hat{v}_{n, s}\right\|_{Y_{s}}+\left\|\nabla \hat{v}_{n, s}\right\|_{Y_{s}} \leq C\left\|D \hat{v}_{n, s}\right\|_{Y_{s}} .
$$

But the left-hand side is one by (3.5) and the right-hand side tends to zero by (3.6), contradicting the assumption that (3.4) fails to hold. Now we have inequality (3.4) for any $\hat{v} \in V(Y)$, wherein $\hat{C}$ does not depend on $\epsilon$.

We remark that we also have the inequality for functions defined only on an open subset $Y_{p} \subset Y$, as long as $Y_{p} \cap Y_{d}$ is not empty. When $Y_{p} \subset Y_{s}$, we can obtain the same inequality provided that we have the boundary condition $\hat{v}=0$ on some positive measure subset of $\partial Y_{p}$.

By the structure of $\Omega$, we can write it as $\Omega=\bigcup_{i \in \mathcal{I}} \epsilon Y^{i}$, where $\epsilon Y^{i}=\left(\epsilon\left(Y+\vec{n}_{i}\right)\right) \cap \Omega$, $\vec{n}_{i}$ is some vector whose components are integers, and $\mathcal{I}$ is some appropriate index set. Let $v \in V_{0}^{\epsilon}(\Omega)$ and define for $y \in Y, \hat{v}^{i}(y)=v\left(\epsilon\left(y+\vec{n}_{i}\right)\right) \in V(Y)$. By our remark above, if $\partial \Omega$ intersects the interior of a cell (i.e., $\Omega$ is not tiled exactly by scaled translates of $Y$ ), the inequality (3.4) still holds on that truncated cell since $v \in V_{0}^{\epsilon}$. Thus (3.4) holds on each $Y^{i}$, so

$$
\left\|\hat{v}_{s}^{i}\right\|_{Y_{s}^{i}}+\left\|\nabla \hat{v}_{s}^{i}\right\|_{Y_{s}^{i}} \leq \hat{C}\left(\left\|D \hat{v}_{s}^{i}\right\|_{Y_{s}^{i}}+\left\|\hat{v}_{s}^{i} \cdot \tau\right\|_{\Gamma^{i}}+\left\|\hat{v}_{d}^{i}\right\|_{Y_{d}^{i}}+\left\|\nabla \cdot \hat{v}^{i}\right\|_{Y^{i}}\right),
$$

and if we sum over all $i$ and make the change of variables $x=\epsilon\left(y+\vec{n}_{i}\right)$ on each $Y^{i}$, we obtain

$$
\epsilon^{-1}\left\|v_{s}\right\|_{\Omega_{s}^{\epsilon}}+\left\|\nabla v_{s}\right\|_{\Omega_{s}^{\epsilon}} \leq C\left(\left\|D v_{s}\right\|_{\Omega_{s}^{\epsilon}}+\epsilon^{-1 / 2}\left\|v_{s} \cdot \tau\right\|_{\Gamma^{\epsilon}}+\epsilon^{-1}\left\|v_{d}\right\|_{\Omega_{d}^{\epsilon}}+\|\nabla \cdot v\|\right),
$$

which gives the desired result $v \in V_{0}^{\epsilon}$.

For $v \in V^{\epsilon}$, as long as $\Omega_{d}$ is not empty, we can adjoin any truncated cell to an entire cell lying beside it (at least for $\epsilon$ small). Since there will be a portion of $\Gamma$ within this new composite cell, we have the inequality (3.4) on the composite cell regardless of the outer boundary conditions. Hence, if $\Omega_{d}$ is not empty, the theorem holds also for all $v \in V^{\epsilon}$.

Proof. [Proof of Theorem 3.1] The theorem follows from the inf-sup theory of saddle point problems $[5,10,11,12]$. For $u, v \in V_{0}^{\epsilon}(\Omega)$ and $w \in W$, we have the bilinear forms

$$
\begin{aligned}
a^{\epsilon}(u, v) & =2 \mu \epsilon^{2}\left(D u_{s}, D v\right)_{\Omega_{s}^{\epsilon}}+\left(\frac{\epsilon \mu \alpha}{\sqrt{K^{\epsilon}}} u_{s} \cdot \tau, v_{s} \cdot \tau\right)_{\Gamma^{\epsilon}}+\left(\mu\left(K^{\epsilon}\right)^{-1} u_{d}, v\right)_{\Omega_{d}^{\epsilon}}, \\
b(v, w) & =(w, \nabla \cdot v)_{\Omega} .
\end{aligned}
$$

Then (3.1)-(3.2) can be rewritten as: Find $\left(u^{\epsilon}, p^{\epsilon}\right) \in V_{0}^{\epsilon} \times W$ such that

$$
\begin{aligned}
a^{\epsilon}\left(u^{\epsilon}, v\right)-b\left(v, p^{\epsilon}\right) & =(f, v), \quad v \in V_{0}^{\epsilon}, \\
b\left(u^{\epsilon}, w\right) & =(q, w), \quad w \in W .
\end{aligned}
$$


We endow $V_{0}^{\epsilon}(\Omega)$ with the norm

$$
\|u\| \|_{\epsilon}=\left(\|u\|^{2}+\|\nabla \cdot u\|^{2}+\epsilon^{2}\left\|\nabla u_{s}\right\|_{\Omega_{s}^{\epsilon}}^{2}\right)^{1 / 2},
$$

for which it is complete. We claim that

$$
\left(\frac{\epsilon \mu \alpha}{\sqrt{K^{\epsilon}}} u_{s} \cdot \tau, v_{s} \cdot \tau\right)_{\Gamma^{\epsilon}} \leq C \epsilon\left\|u_{s} \cdot \tau\right\|_{\Gamma^{\epsilon}}\left\|v_{s} \cdot \tau\right\|_{\Gamma^{\epsilon}} \leq C \mid\|u\|\left\|_{\epsilon}\right\|\|v\| \|_{\epsilon}
$$

so that that both $a^{\epsilon}$ and $b$ are bounded (i.e., continuous) with constants independent of $\epsilon$. To see the claim, compute

$$
\begin{aligned}
\epsilon\left\|u_{s} \cdot \tau\right\|_{\Gamma^{\epsilon}}^{2} & =\epsilon \sum_{i \in \mathcal{I}}\left\|u_{s} \cdot \tau\right\|_{\epsilon \Gamma^{i}}^{2} \\
& =\epsilon \sum_{i \in \mathcal{I}} \epsilon\left\|\hat{u}_{s} \cdot \hat{\tau}\right\|_{\Gamma^{i}}^{2} \\
& \leq \epsilon^{2} \sum_{i \in \mathcal{I}} \hat{C}\|\hat{u}\|_{Y_{s}^{i}}\|\hat{u}\|_{H^{1}\left(Y_{s}^{i}\right)} \\
& \leq \hat{C} \epsilon \sum_{i \in \mathcal{I}}\|u\|_{\epsilon Y_{s}^{i}}\|u\|_{H^{1}\left(\epsilon Y_{s}^{i}\right)} \\
& \leq \frac{\hat{C}}{2} \sum_{i \in \mathcal{I}}\left[\|u\|_{\epsilon Y_{s}^{i}}^{2}+\epsilon^{2}\|u\|_{H^{1}\left(\epsilon Y_{s}^{i}\right)}^{2}\right] \\
& =\frac{\hat{C}}{2}\left[\|u\|_{\Omega_{s}^{\epsilon}}^{2}+\epsilon^{2}\|u\|_{H^{1}\left(\Omega_{s}^{\epsilon}\right)}^{2}\right] \\
& \leq C\|u\| \|_{\epsilon}^{2} .
\end{aligned}
$$

Moreover, $a^{\epsilon}$ is coercive on $V_{0}^{\epsilon} \cap\left\{v \in V_{0}^{\epsilon}: \nabla \cdot v=0\right\}$ by Lemma 3.2, with bound independent of $\epsilon$.

It remains to show the inf-sup condition, but this follows from the corresponding condition known for the Stokes system on $\Omega$; that is,

$$
\begin{aligned}
\inf _{w \in W} \sup _{v \in V_{0}^{\epsilon}} \frac{(\nabla \cdot v, w)}{\|v\|\left\|_{\epsilon}\right\| w \|} & \geq \inf _{w \in W} \sup _{v \in\left(H_{0}^{1}\right)^{2}} \frac{(\nabla \cdot v, w)}{\|v\|\left\|_{\epsilon}\right\| w \|} \\
& \geq \inf _{w \in W} \sup _{v \in\left(H_{0}^{1}\right)^{2}} \frac{(\nabla \cdot v, w)}{2\|v\|_{\left(H^{1}(\Omega)\right)^{2}}\|w\|} \geq \gamma>0,
\end{aligned}
$$

for some $\gamma$ independent of $\epsilon$, since $\left(H_{0}^{1}\right)^{2} \subset V_{0}^{\epsilon}$ and $2\|v\|_{\left(H^{1}(\Omega)\right)^{2}} \geq\|\| v\|\|_{\epsilon}$.

Now the inf-sup theory provides the existence and uniqueness of a solution to our system (3.7)-(3.8) [12]. Moreover,

$$
\left\|u^{\epsilon} \mid\right\|_{\epsilon}+\left\|p^{\epsilon}\right\| \leq C(\|f\|+\|q\|),
$$

where $C$ depends on $\gamma$, the coercivity bound for $a^{\epsilon}$, and the continuity bounds for $a^{\epsilon}$ and $b$, each of which is independent of $\epsilon$. Finally, (3.11) and (3.10) imply (3.3).

4. Two-Scale Convergence Results for Bimodal Media. In this section we make note of some slight extensions of the two-scale convergence results of Allaire [1, 14]. Lemmas 4.1 and 4.4 can be deduced easily from the proof of Theorem 2.7 in [1]. We include the following statements and proofs for clarity and completeness. We 
first recall that $\mathcal{D}\left(\Omega ; C_{\#}^{\infty}(Y)\right)$ is the set of infinitely differentiable functions in $\Omega \times Y$ that have compact support in $\Omega$ and are periodic in $Y$, and we recall the following definition.

DEFinition 4.1. If $\left\{u^{\epsilon}\right\}_{\epsilon} \subset L^{2}(\Omega)$ and $u_{0}(x, y) \in L^{2}(\Omega \times Y)$ are such that

$$
\lim _{\epsilon \rightarrow 0} \int_{\Omega} u^{\epsilon}(x) \phi(x, x / \epsilon) d x=\frac{1}{|Y|} \int_{\Omega} \int_{Y} u_{0}(x, y) \phi(x, y) d y d x
$$

for any function $\phi \in \mathcal{D}\left(\Omega ; C_{\#}^{\infty}(Y)\right)$, then $\left\{u^{\epsilon}\right\}_{\epsilon}$ is said to two-scale converge in $\Omega \times Y$ to $u_{0}(x, y)$, and we write this as

$$
u^{\epsilon} \longrightarrow u_{0} \quad \text { in } \Omega \times Y \text { as } \epsilon \rightarrow 0 .
$$

LEMMA 4.1. Let $\ell=s$ or $d$ and $\chi_{\ell}^{\epsilon}$ be the characteristic function on $\Omega_{\ell}^{\epsilon}$. If $u^{\epsilon}$ is such that $\left\|u^{\epsilon}\right\| \leq C$ for some constant $C$ independent of $\epsilon$, then a subsequence of $\chi_{\ell}^{\epsilon} u^{\epsilon}$ two-scale converges to some $\psi_{0}^{\ell} \in L^{2}(\Omega \times Y)$ such that $\operatorname{supp}\left(\psi_{0}^{\ell}\right) \subset \Omega \times \bar{Y}_{\ell}$. Moreover, if $u^{\epsilon}$ two-scale converges to $u_{0} \in L^{2}(\Omega \times Y)$, then $\left.\chi_{\ell}^{\epsilon} u_{\epsilon} \longrightarrow u_{0}\right|_{\Omega \times Y_{\ell}}$ in $\Omega \times Y_{\ell}$.

Proof. Because $\left\|\chi_{\ell}^{\epsilon} u^{\epsilon}\right\| \leq\left\|u^{\epsilon}\right\| \leq C$, a subsequence of $\chi_{\ell}^{\epsilon} u^{\epsilon}$ two-scale converges to some $\psi_{0}^{\ell} \in L^{2}(\Omega \times Y)[1]$. Take a test function $\phi \in \mathcal{D}\left(\Omega ; C_{\#}^{\infty}(Y)\right)$ supported in $\Omega \times Y_{k}$ where $k \neq \ell$. Then $\phi^{\epsilon}(x)=\phi(x, x / \epsilon)$ is supported in $\Omega_{k}^{\epsilon}$ and

$$
0=\lim _{\epsilon \rightarrow 0} \int_{\Omega} \chi_{\ell}^{\epsilon} u^{\epsilon} \phi^{\epsilon} d x=\frac{1}{|Y|} \int_{\Omega} \int_{Y} \psi_{0}^{\ell} \phi d y d x=\frac{1}{|Y|} \int_{\Omega} \int_{Y_{k}} \psi_{0}^{\ell} \phi d y d x
$$

This holds for all such $\phi$, so $\psi_{0}^{\ell}=0$ on $\Omega \times Y_{k}$.

Now, take a test function $\phi \in \mathcal{D}\left(\Omega ; C_{\#}^{\infty}(Y)\right)$ with support in $\Omega \times Y_{\ell}$. Then

$$
\int_{\Omega} \chi_{\ell}^{\epsilon} u^{\epsilon} \phi^{\epsilon} d x=\int_{\Omega} u^{\epsilon} \phi^{\epsilon} d x
$$

so, taking the limit as $\epsilon \rightarrow 0$,

$$
\frac{1}{|Y|} \int_{\Omega} \int_{Y_{\ell}} \psi_{0}^{\ell} \phi d y d x=\frac{1}{|Y|} \int_{\Omega} \int_{Y} u_{0} \phi d y d x
$$

An application of Lusin's Theorem completes the lemma.

Lemma 4.1 allows us to make the following definition and gives the following corollary. Note that a function in $\mathcal{D}\left(\Omega ; C_{\#}^{\infty}\left(Y_{\ell}\right)\right)$ is considered to be only $Y$-periodic in $y$, with no condition imposed on $\Gamma$.

Definition 4.2. If $\ell=s$ or $d$ and $\left\{u_{\ell}^{\epsilon}\right\}_{\epsilon} \subset L^{2}\left(\Omega_{\ell}^{\epsilon}\right)$ is such that, for any function $\phi(x, y)$ in $\mathcal{D}\left(\Omega ; C_{\#}^{\infty}\left(Y_{\ell}\right)\right)$,

$$
\lim _{\epsilon \rightarrow 0} \int_{\Omega_{\ell}^{\epsilon}} u_{\ell}^{\epsilon}(x) \phi(x, x / \epsilon) d x=\frac{1}{|Y|} \int_{\Omega} \int_{Y_{\ell}} u_{0}(x, y) \phi(x, y) d y d x
$$

for some $u_{0}(x, y)$ in $L^{2}\left(\Omega \times Y_{\ell}\right)$, then $\left\{u_{\ell}^{\epsilon}\right\}_{\epsilon}$ is said to two-scale converge in $\Omega \times Y_{\ell}$ to $u_{0}(x, y)$ as $\epsilon \rightarrow 0$.

COROLlary 4.2. If $u_{\ell}^{\epsilon} \in L^{2}\left(\Omega_{\ell}^{\epsilon}\right)$ and there exists $C>0$ such that $\left\|u_{\ell}^{\epsilon}\right\|_{\Omega_{\ell}^{\epsilon}} \leq C$ for all $\epsilon>0$, then there exists a subsequence which two-scale converges in $\Omega \times Y_{\ell}$ to $u_{0} \in L^{2}\left(\Omega \times Y_{\ell}\right)$.

The following lemma is immediate and illuminates the connection between weak and two-scale convergence. 
LEMmA 4.3. If $\left\{u_{\ell}^{\epsilon}\right\}_{\epsilon}$ two-scale converges to $u_{0}(x, y)$ in $\Omega \times Y_{\ell}$, and $\hat{u}_{\ell}^{\epsilon}$ denotes the extension of $u_{\ell}^{\epsilon}$ by zero to $\Omega$, then $\hat{u}_{\ell}^{\epsilon}$ converges weakly to $\frac{1}{|Y|} \int_{Y_{\ell}} u_{0}(x, y) d y$ in $L^{2}(\Omega)$.

The next results will be needed to prove our homogenization result. As usual, $H_{\#}^{1}(Y)$ denotes the $Y$-periodic functions in $H^{1}(Y)$.

LEMMA 4.4. Fix $\ell=s$ or $d$.

(a) If $\left\|u_{\ell}^{\epsilon}\right\|_{\Omega_{\ell}^{\epsilon}} \leq C$ and $\left\|\epsilon \nabla u_{\ell}^{\epsilon}\right\|_{\Omega_{\ell}^{\epsilon}} \leq C$ for some constant $C$, then there exists $u_{0, \ell} \in\left(L^{2}\left(\Omega ; H_{\#}^{1}\left(Y_{\ell}\right)\right)\right)^{2}$ such that some subsequence of $\left\{u_{\ell}^{\epsilon}\right\}_{\epsilon}$ two-scale converges in $\Omega \times Y_{\ell}$ to $u_{0, \ell}$ and $\left\{\epsilon \nabla u_{\ell}^{\epsilon}\right\}_{\epsilon}$ two-scale converges in $\Omega \times Y_{\ell}$ to $\nabla_{y} u_{0, \ell}$.

(b) If $\left\|u_{\ell}^{\epsilon}\right\|_{\Omega_{\ell}^{\epsilon}} \leq C$ and $\left\|\nabla \cdot u_{\ell}^{\epsilon}\right\|_{\Omega_{\ell}^{\epsilon}} \leq C$ for some constant $C$, then there exists $u_{0, \ell} \in\left(L^{2}\left(\Omega ; H\left(\operatorname{div}, Y_{\ell}\right)\right)\right)^{2}$ such that some subsequence of $\left\{u_{\ell}^{\epsilon}\right\}_{\epsilon}$ two-scale converges in $\Omega \times Y_{\ell}$ to $u_{0, \ell}$ and $\left\{\nabla \cdot u_{\ell}^{\epsilon}\right\}_{\epsilon}$, extended to $\Omega$ by zero, converges weakly in $L^{2}(\Omega)$ to $\frac{1}{|Y|} \int_{Y_{\ell}} \nabla_{x} \cdot u_{0, \ell} d y$. Moreover, $\nabla_{y} \cdot u_{0, \ell}=0$.

Proof. For result (a), by Corollary 4.2, we have for some subsequence both

$$
\begin{aligned}
& u_{\ell}^{\epsilon} \longrightarrow u_{0, \ell} \quad \text { in } \Omega \times Y_{\ell}, \\
& \epsilon \nabla u_{\ell}^{\epsilon} \longrightarrow \psi_{0, \ell} \quad \text { in } \Omega \times Y_{\ell} .
\end{aligned}
$$

Let $\phi \in\left(\mathcal{D}\left(\Omega ; C_{\#}^{\infty}\left(Y_{\ell}\right)\right)\right)^{2}$ be such that $\left.\phi\right|_{\Omega \times \Gamma}=0$, and let $\phi^{\epsilon}(x)=\phi(x, x / \epsilon)$. Compute

$$
\left(\epsilon \nabla u_{\ell}^{\epsilon}, \phi^{\epsilon}\right)_{\Omega_{\ell}^{\epsilon}}=-\left(u_{\ell}^{\epsilon}, \epsilon \nabla_{x} \cdot \phi^{\epsilon}+\nabla_{y} \cdot \phi^{\epsilon}\right)_{\Omega_{\ell}^{\epsilon}},
$$

so that as $\epsilon \rightarrow 0$,

$$
\left(\psi_{0, \ell}, \phi\right)_{\Omega \times Y_{\ell}}=-\left(u_{0, \ell}, \nabla_{y} \cdot \phi\right)_{\Omega \times Y_{\ell}}=\left(\nabla_{y} u_{0, \ell}, \phi\right)_{\Omega \times Y_{\ell}}-\left(u_{0, \ell} \cdot \eta, \phi\right)_{\Omega \times\left(\partial Y_{\ell} \backslash \Gamma\right)} .
$$

With $\left.\phi\right|_{\Omega \times \partial Y_{\ell}}=0$, we conclude that $\psi_{0, \ell}=\nabla_{y} u_{0, \ell}$. Then we further conclude that $u_{0, \ell}$ is periodic in $y \in Y_{\ell}$, i.e., that $u_{0, \ell} \in\left(L^{2}\left(\Omega ; H_{\#}^{1}\left(Y_{\ell}\right)\right)\right)^{2}$.

For (b), Corollary 4.2 gives us two-scale convergence of $u_{\ell}^{\epsilon}$ to $u_{0, \ell}$, and then weak convergence of $\nabla \cdot u_{\ell}^{\epsilon}$ to $\frac{1}{|Y|} \int_{Y_{\ell}} \nabla_{x} \cdot u_{0, \ell} d y$ follows easily. To obtain $\nabla_{y} \cdot u_{0, \ell}=0$, note that for $\phi \in \mathcal{D}\left(\Omega ; C_{0}^{\infty}(Y)\right)$,

$$
\int_{\Omega_{\ell}^{\epsilon}} \nabla \cdot u_{\ell}^{\epsilon} \phi^{\epsilon} d x=-\int_{\Omega_{\ell}^{\epsilon}} u_{\ell}^{\epsilon} \cdot\left(\nabla_{x} \phi^{\epsilon}+\epsilon^{-1} \nabla_{y} \phi^{\epsilon}\right) d x,
$$

By Corollary 4.2, the left-hand side and the first term on the right-hand side both converge as $\epsilon \rightarrow 0$. Thus we obtain

$$
\lim _{\epsilon \rightarrow 0} \int_{\Omega_{\ell}^{\epsilon}} u_{\ell}^{\epsilon} \cdot \nabla_{y} \phi d x=0
$$

which implies that $\nabla_{y} \cdot u_{0, \ell}=0$.

LEMMA 4.5. If $u_{s}^{\epsilon}$ is such that $\left\|u_{s}^{\epsilon}\right\|_{\Omega_{s}^{\epsilon}}$ and $\left\|\epsilon \nabla u_{s}^{\epsilon}\right\|_{\Omega_{s}^{\epsilon}}$ are bounded independent of $\epsilon$ and $\Gamma^{\epsilon}$ is a smooth submanifold of $\Omega^{\epsilon}$, then for $\phi \in\left(\mathcal{D}\left(\Omega ; C_{\#}^{\infty}\left(Y_{s}\right)\right)\right)^{2}$,

$$
\lim _{\epsilon \rightarrow 0} \epsilon\left(u_{s}^{\epsilon} \cdot \tau, \phi \cdot \tau\right)_{\Gamma^{\epsilon}}=|Y|^{-1}\left(u_{0, s} \cdot \tau, \phi \cdot \tau\right)_{\Omega \times \Gamma}
$$


where $u_{0, s}$ is the two-scale limit of $u_{s}^{\epsilon}$ in $\Omega \times Y_{s}$. Moreover,

$$
\lim _{\epsilon \rightarrow 0} \epsilon\left(u_{s}^{\epsilon} \cdot \eta_{s}, \phi \cdot \eta_{s}\right)_{\Gamma^{\epsilon}}=|Y|^{-1}\left(u_{0, s} \cdot \eta_{s}, \phi \cdot \eta_{s}\right)_{\Omega \times \Gamma} .
$$

Proof. Let Let $\Phi \in\left(\mathcal{D}\left(\Omega ; C_{\#}^{\infty}\left(Y_{s}\right)\right)\right)^{2 \times 2}$ and $\Phi^{\epsilon}(x)=\Phi(x, x / \epsilon)$. Then

$$
\left(\epsilon \nabla u_{s}^{\epsilon}, \Phi^{\epsilon}\right)_{\Omega_{s}^{\epsilon}}=-\epsilon\left(u_{s}^{\epsilon}, \nabla_{x} \cdot \Phi^{\epsilon}\right)_{\Omega_{s}^{\epsilon}}-\left(u_{s}^{\epsilon}, \nabla_{y} \cdot \Phi^{\epsilon}\right)_{\Omega_{s}^{\epsilon}}+\epsilon\left(u_{s}^{\epsilon}, \Phi^{\epsilon} \cdot \eta_{s}\right)_{\Gamma^{\epsilon}} .
$$

Taking the limit of both sides as $\epsilon \rightarrow 0$, we obtain from Lemma 4.4

$$
|Y|^{-1}\left(\nabla_{y} u_{0, s}, \Phi\right)_{\Omega \times Y_{s}}=-|Y|^{-1}\left(u_{0, s}, \nabla_{y} \cdot \Phi\right)_{\Omega \times Y_{s}}+\lim _{\epsilon \rightarrow 0} \epsilon\left(u_{s}^{\epsilon}, \Phi^{\epsilon} \cdot \eta_{s}\right)_{\Gamma^{\epsilon}} .
$$

This implies that

$$
|Y|^{-1}\left(u_{0, s}, \Phi \cdot \eta_{s}\right)_{\Omega \times \Gamma}=\lim _{\epsilon \rightarrow 0} \epsilon\left(u_{s}^{\epsilon}, \Phi^{\epsilon} \cdot \eta_{s}\right)_{\Gamma^{\epsilon}}
$$

Since $\Gamma$ is smooth, the Tubular Neighborhood Theorem from topology allows us to extend the normal vector field $\eta_{s}$ on $\Gamma$ to a smooth vector field $\hat{N}(y)$ on $Y$. If we do this locally, and patch the results together using a partition of unity argument, we can obtain smooth vector fields after periodic extension. We can then define $N^{\epsilon}$ and $T^{\epsilon}$ on all of $\Omega$ by setting $N^{\epsilon}(x)=N(x / \epsilon)=\hat{N}(y)$ and $T^{\epsilon}(x)=T(x / \epsilon)=\hat{T}(y)$.

Now take $\Phi=T^{\epsilon}\left(\phi \cdot T^{\epsilon}\right)\left(N^{\epsilon}\right)^{T}$ in (4.1), where $\phi \in\left(\mathcal{D}\left(\Omega ; C_{\#}^{\infty}\left(Y_{s}\right)\right)\right)^{2}$. This yields the first result. Replacing $T^{\epsilon}$ with $N^{\epsilon}$ gives the second result.

In fact, the following more general definition makes sense and was previously stated in [21], [2], and [9].

Definition 4.3. If $\ell=s$ or $d$ and $\left\{u_{\ell}^{\epsilon}\right\}_{\epsilon} \subset L^{2}\left(\Gamma^{\epsilon}\right)$ is such that, for any function $\phi(x, y)$ in $\mathcal{D}\left(\Omega ; C_{\#}^{\infty}(\Gamma)\right)$ which is $Y$-periodic in $y$,

$$
\lim _{\epsilon \rightarrow 0} \int_{\Gamma^{\epsilon}} u_{\ell}^{\epsilon}(x) \phi(x, x / \epsilon) d x=\frac{1}{|Y|} \int_{\Omega} \int_{\Gamma} u_{0}(x, y) \phi(x, y) d S d x
$$

for some $u_{0}(x, y)$ in $L^{2}(\Omega \times \Gamma)$, then $\left\{u_{\ell}^{\epsilon}\right\}_{\epsilon}$ is said to two-scale converge on $\Omega \times \Gamma$ to $u_{0}(x, y)$ as $\epsilon \rightarrow 0$.

5. Proof of the Homogenization Result. We now prove rigorously the homogenization results obtained formally in Section 2. In the first theorem, we obtain only weak convergence in $H(\operatorname{div}, \Omega) \times W$ to the solution of the homogenized problem. In the second theorem, we show that in fact we have strong convergence of $\left(u^{\epsilon}, p^{\epsilon}\right)$ in $L^{2}(\Omega) \times W$.

THEOREM 5.1. There exists $(u, p) \in H(\operatorname{div}, \Omega) \times W$ such that the velocity $u^{\epsilon}$ converges weakly to $u$ in $H(\operatorname{div}, \Omega), p^{\epsilon}$ converges weakly to $p$ in $W$, and $(u, p)$ is the unique solution to the homogenized Darcy problem

$$
\begin{array}{rlrl}
\mu \tilde{K}^{-1} u+\nabla p & =f & & \text { in } \Omega, \\
\nabla \cdot u=q & & \text { in } \Omega, \\
u \cdot \eta=0 & & \text { on } \partial \Omega,
\end{array}
$$

where the tensor $\tilde{K}$ is defined by (2.13) and (2.4)-(2.10).

Proof. By our energy estimates in Theorem 3.1 and the two-scale convergence results of $[1,14]$, Corollary 4.2 , and Lemma 4.4 , for $\ell=s, d$, there exists $p_{0}(x, y) \in$ 
$L^{2}(\Omega \times Y)$ and $u_{0}(x, y) \in L^{2}(\Omega \times Y)$ such that the following two-scale convergences hold:

$$
\begin{array}{cl}
p^{\epsilon} \longrightarrow p_{0} & \text { in } \Omega \times Y, \\
u^{\epsilon} \longrightarrow u_{0} & \text { in } \Omega \times Y, \\
u_{\ell}^{\epsilon} \longrightarrow u_{0, \ell} & \text { in } \Omega \times Y_{\ell}, \\
\epsilon \nabla u_{s}^{\epsilon} \longrightarrow \nabla_{y} u_{0, s} & \text { in } \Omega \times Y_{s} .
\end{array}
$$

Moreover, $u_{0, s} \in\left(L^{2}\left(\Omega ; H_{\#}^{1}\left(Y_{s}\right)\right)\right)^{2}$,

$$
\nabla \cdot u^{\epsilon}=q
$$

and

$$
\nabla_{y} \cdot u_{0}(x, y)=0 .
$$

Let $\Psi(x, y) \in C_{0}^{\infty}(\Omega \times Y)$ be $Y$-periodic. Take $v(x)=\epsilon \Psi(x, x / \epsilon)$ in the variational problem (3.1), so that

$$
\left(p^{\epsilon}, \nabla_{y} \cdot \Psi\right)+O(\epsilon)=0 .
$$

As $\epsilon \rightarrow 0$, we obtain

$$
\left(p_{0}, \nabla_{y} \cdot \Psi\right)_{\Omega \times Y}=0 .
$$

This implies that $\nabla_{y} p_{0}=0$, so that $p_{0}(x, y)=p_{0}(x)$ only.

Next take $\Psi \in\left(\mathcal{D}\left(\Omega ; C_{\#}^{\infty}(Y)\right)\right)^{2}$ with $\nabla_{y} \cdot \Psi=0$, and let $v(x)=\Psi(x, x / \epsilon)$ in the variational equation (3.1), so that

$$
\begin{aligned}
2 \mu \epsilon^{2}\left(D u_{s}^{\epsilon}, D \Psi^{\epsilon}\right)_{\Omega_{s}^{\epsilon}} & +\left(\frac{\epsilon \mu \alpha}{\sqrt{K^{\epsilon}}} u_{s}^{\epsilon} \cdot \tau, \Psi^{\epsilon} \cdot \tau\right)_{\Gamma^{\epsilon}}+\left(\mu\left(K^{\epsilon}\right)^{-1} u_{d}^{\epsilon}, \Psi^{\epsilon}\right)_{\Omega_{d}^{\epsilon}} \\
-\left(p^{\epsilon}, \nabla_{x} \cdot \Psi^{\epsilon}\right) & =\left(f, \Psi^{\epsilon}\right) .
\end{aligned}
$$

Using Lemma 4.5, passing to the two-scale limit gives

$$
\begin{aligned}
& 2 \mu\left(D_{y} u_{0, s}, D_{y} \Psi\right)_{\Omega \times Y_{s}}+\left(\frac{\mu \alpha}{\sqrt{K}} u_{0, s} \cdot \tau, \Psi \cdot \tau\right)_{\Omega \times \Gamma}+\left(\mu K^{-1} u_{0, d}, \Psi\right)_{\Omega \times Y_{d}} \\
& -\left(p_{0}, \nabla_{x} \cdot \Psi\right)_{\Omega \times Y}=(f, \Psi)_{\Omega \times Y} .
\end{aligned}
$$

Integrating by parts and collecting terms, we obtain

$$
\begin{aligned}
& \left(-2 \mu \nabla_{y} \cdot D_{y} u_{0, s}+\nabla_{x} p_{0}-f, \Psi\right)_{\Omega \times Y_{s}}+\left(\mu K^{-1} u_{0, d}+\nabla_{x} p_{0}-f, \Psi\right)_{\Omega \times Y_{d}} \\
& \quad+\left(\frac{\mu \alpha}{\sqrt{K}} u_{0, s} \cdot \tau+2 \mu \tau \cdot D_{y} u_{0, s} \cdot \eta_{s}, \Psi \cdot \tau\right)_{\Omega \times \Gamma} \\
& \quad+\left(2 \mu \eta_{s} \cdot D_{y} u_{0, s} \cdot \eta_{s}, \Psi \cdot \eta_{s}\right)_{\Omega \times \Gamma}=0 .
\end{aligned}
$$

It is a well-known result that if $(\Phi, \Psi)_{\Omega \times Y}=0$, with $\nabla_{y} \cdot \Psi=0$ and $\Psi \in$ $\mathcal{D}\left(\Omega ; C_{0}^{\infty}(Y)\right)^{2}$, then $\Phi=\nabla_{y} \psi$ for some $\psi \in L^{2}\left(\Omega ; H^{1}(Y)\right)[28]$. Restrict to $\Psi \in$ $\left(\mathcal{D}\left(\Omega ; C_{0}^{\infty}\left(Y_{s}\right)\right)\right)^{2}$ to obtain

$$
\left(-2 \mu \nabla_{y} \cdot D_{y} u_{0, s}+\nabla_{x} p_{0}-f, \Psi\right)_{\Omega \times Y_{s}}=0 .
$$


Thus, there exists $p_{1, s}(x, y) \in L^{2}\left(\Omega ; H^{1}\left(Y_{s}\right)\right)$ such that in $\Omega \times Y_{s}$,

$$
-2 \mu \nabla_{y} \cdot D_{y} u_{0, s}+\nabla_{x} p_{0}-f=-\nabla_{y} p_{1, s} .
$$

Likewise, we obtain $p_{1, d}(x, y) \in L^{2}\left(\Omega ; H^{1}\left(Y_{d}\right)\right)$ such that in $\Omega \times Y_{d}$,

$$
K^{-1} u_{0, d}+\nabla_{x} p_{0}-f=-\nabla_{y} p_{1, d} .
$$

Thus for all $\Psi \in \mathcal{D}\left(\Omega ; C_{\#}^{\infty}(Y)\right)^{2}$ satisfying the divergence constraint,

$$
\begin{aligned}
& \left(-\nabla_{y} p_{1, s}, \Psi\right)_{\Omega \times Y_{s}}+\left(-\nabla_{y} p_{1, d}, \Psi\right)_{\Omega \times Y_{d}} \\
& \quad+\left(\frac{\mu \alpha}{\sqrt{K}} u_{0, s} \cdot \tau+2 \mu \tau \cdot D_{y} u_{0, s} \cdot \eta_{s}, \Psi \cdot \tau\right)_{\Omega \times \Gamma} \\
& \quad+\left(2 \mu \eta_{s} \cdot D_{y} u_{0, s} \cdot \eta_{s}, \Psi \cdot \eta_{s}\right)_{\Omega \times \Gamma}=0,
\end{aligned}
$$

so integrating by parts yields

$$
\begin{aligned}
& \left(\frac{\mu \alpha}{\sqrt{K}} u_{0, s} \cdot \tau+2 \mu \tau \cdot D_{y} u_{0, s} \cdot \eta_{s}, \Psi \cdot \tau\right)_{\Omega \times \Gamma} \\
& \quad+\left(2 \mu \eta_{s} \cdot D_{y} u_{0, s} \cdot \eta_{s}-p_{1, s}+p_{1, d}, \Psi \cdot \eta_{s}\right)_{\Omega \times \Gamma}=0 .
\end{aligned}
$$

By noting for example that there exists a weak solution (see [28] or the proof of Theorem 3.1) $\Psi \in L^{2}\left(\Omega ;\left(H_{\#}^{1}(Y)\right)^{2}\right), w \in L^{2}\left(\Omega ; L^{2}(Y) / \mathbb{R}\right)$ to

$$
\begin{aligned}
-\Delta_{y} \Psi+\nabla_{y} w & =0 & & \text { on } \Omega \times Y_{s}, \\
\nabla_{y} \cdot \Psi & =0 & & \text { on } \Omega \times Y_{s}, \\
\Psi \cdot \eta_{s} & =0 & & \text { on } \Omega \times \Gamma, \\
\Psi \cdot \tau & =\frac{\mu \alpha}{\sqrt{K}} u_{0, s} \cdot \tau+2 \mu \tau \cdot D_{y} u_{0, s} \cdot \eta_{s} & & \text { on } \Omega \times \Gamma, \\
\Psi & =0 & & \text { on } \Omega \times\left(\partial Y_{s} \backslash \Gamma\right),
\end{aligned}
$$

and by the fact that $\mathcal{D}\left(\Omega ; C_{\#}^{\infty}(Y)\right)$ is dense in $L^{2}\left(\Omega ; H_{\#}^{1}(Y)\right)$, we obtain that each individual term in (5.11) vanishes. We then finally obtain that the two-scale variational equations (5.9) and (5.10) are equivalent to

$$
\begin{aligned}
-2 \mu \nabla_{y} \cdot D_{y} u_{0, s}+\nabla_{x} p_{0}(x)+\nabla_{y} p_{1, s}(x, y) & =f & & \text { in } \Omega \times Y_{s}, \\
\nabla_{y} \cdot u_{0, s} & =0 & & \text { in } \Omega \times Y_{s}, \\
\mu K^{-1} u_{0, d}+\nabla_{x} p_{0}(x)+\nabla_{y} p_{1, d}(x, y) & =f & & \text { in } \Omega \times Y_{d}, \\
\nabla_{y} \cdot u_{0, d} & =0 & & \text { in } \Omega \times Y_{d}, \\
2 \mu \eta_{s} \cdot D_{y} u_{0, s} \cdot \eta_{s} & =p_{1, s}-p_{1, d} & & \text { on } \Omega \times \Gamma, \\
2 \tau \cdot D_{y} u_{0, s} \cdot \eta_{s} & =-\frac{\alpha}{\sqrt{K}} u_{0, s} \cdot \tau & & \text { on } \Omega \times \Gamma .
\end{aligned}
$$

Let $\left(\omega_{j}^{s}, \Phi_{j}^{s}\right)$ and $\left(\omega_{j}^{d}, \Phi_{j}^{d}\right)$ be $Y$-periodic solutions to the auxiliary problem on $Y_{s}$ and $Y_{d}$ given in (2.4)-(2.10). Then, because the above problem has a unique solution, it is clear that we can express $u_{0, s}$ and $u_{0, d}$ as in (2.11):

$$
u_{0, \ell}(x, y)=\frac{1}{\mu} \sum_{j=1}^{N}\left(f_{j}(x)-\frac{\partial p_{0}}{\partial x_{j}}(x)\right) \omega_{j}^{\ell}(y),
$$


where $\ell=s$ or $d$. Averaging over $Y_{s}$ and $Y_{d}$ as in (2.12), we get

$$
\begin{aligned}
\bar{u}_{0} & =\bar{u}_{0, s}+\bar{u}_{0, d} \\
& =\frac{1}{\mu|Y|} \sum_{j=1}^{N}\left(f_{j}(x)-\partial_{x_{j}} p_{0}(x)\right)\left(\int_{Y_{s}} \omega_{j}^{s}(y)+\int_{Y_{d}} \omega_{j}^{d}(y)\right) \\
& =\frac{\tilde{K}}{\mu}\left(f-\nabla p_{0}\right)
\end{aligned}
$$

where $\tilde{K}$ is as in $(2.13)$.

By Lemma 4.3 and (5.8), we obtain the weak convergence results in $L^{2}(\Omega)$

$$
\begin{aligned}
& p^{\epsilon} \rightarrow \frac{1}{|Y|} \int_{Y} p_{0}(x, y) d y=p_{0}(x), \\
& u^{\epsilon} \rightarrow \frac{1}{|Y|} \int_{Y} u_{0}(x, y) d y=\bar{u}_{0}, \\
& q=\nabla \cdot u^{\epsilon} \rightarrow \nabla \cdot \bar{u}_{0}=q .
\end{aligned}
$$

Setting $p=p_{0}$ and $u=\bar{u}_{0},(5.18)-(5.21)$ gives the theorem, since convergence on the boundary of the domain is trivial.

ThEOREM 5.2. Let $\left(u_{0}, p_{0}\right) \in L^{2}(\Omega \times Y) \times W$ be the two scale limit of $\left(u^{\epsilon}, p^{\epsilon}\right)$ as before. Then $u^{\epsilon}-u_{0}^{\epsilon}$ converges to 0 strongly in $L^{2}(\Omega)$, where $u_{0}^{\epsilon}=u(x, x / \epsilon)$, and $p^{\epsilon}$ converges strongly to $p_{0}(x)$ in $W$.

Proof. Since $\left(\bar{u}_{0}, p_{0}\right)$ satisfies (5.1)-(5.2) and $\mu$ and $\tilde{K}$ are smooth, if $f \in H^{1}(\Omega)$ and $q \in L^{2}$, then $p_{0} \in H^{2}(\Omega)$. It follows that $\bar{u}_{0} \in H^{1}(\Omega)$ and $\nabla_{x} u_{0}(x, y) \in L^{2}(\Omega)$. We also know that $\nabla_{y} u_{0}(x, y)=0 \in L^{2}\left(\Omega \times Y_{s}\right)$, so $u_{0}(x, x / \epsilon) \in V^{\epsilon}$. Since $\mathcal{D}(\Omega)$ is dense in $V^{\epsilon}$, we can use $u_{0}^{\epsilon}$ as a two-scale test function. By two-scale convergence

$$
\lim _{\epsilon \rightarrow 0} a^{\epsilon}\left(u^{\epsilon}, u_{0}^{\epsilon}\right)=\bar{a}\left(u_{0}, u_{0}\right)
$$

where

$$
\bar{a}(v, z)=2 \mu\left(D_{y} v, D_{y} z\right)_{\Omega \times Y_{s}}+\left(\frac{\mu \alpha}{\sqrt{K}} v_{s} \cdot \tau, z_{s} \cdot \tau\right)_{\Omega \times \Gamma}+\left(\mu K^{-1} v, z\right)_{\Omega \times Y_{d}} .
$$

Similarly,

$$
\lim _{\epsilon \rightarrow 0} a^{\epsilon}\left(u_{0}^{\epsilon}, u_{0}^{\epsilon}\right)=\bar{a}\left(u_{0}, u_{0}\right)
$$

by two-scale convergence. Subtracting, we obtain $\lim _{\epsilon \rightarrow 0} a^{\epsilon}\left(u^{\epsilon}-u_{0}^{\epsilon}, u_{0}^{\epsilon}\right)=0$.

We can also show that $\lim _{\epsilon \rightarrow 0} a^{\epsilon}\left(u^{\epsilon}, u^{\epsilon}\right)=\bar{a}\left(u_{0}, u_{0}\right)$. Since

$$
\begin{aligned}
a^{\epsilon}\left(u^{\epsilon}, u^{\epsilon}\right)-b\left(u^{\epsilon}, p^{\epsilon}\right) & =\left(f, u^{\epsilon}\right), \\
b\left(u^{\epsilon}, p^{\epsilon}\right) & =\left(q, p^{\epsilon}\right),
\end{aligned}
$$

we know that $a^{\epsilon}\left(u^{\epsilon}, u^{\epsilon}\right)=\left(f, u^{\epsilon}\right)_{\Omega^{\epsilon}}+\left(q, p^{\epsilon}\right)_{\Omega^{\epsilon}}$. Taking the limit of both sides as $\epsilon \rightarrow 0$, by our weak convergence results in Theorem 5.1 , the right hand side converges to $\left(f, \bar{u}_{0}\right)_{\Omega}+\left(q, p_{0}\right)_{\Omega}$. By $(5.2), q=\nabla \cdot \bar{u}_{0}$, so integrating by parts gives that

$$
\lim _{\epsilon \rightarrow 0} a^{\epsilon}\left(u^{\epsilon}, u^{\epsilon}\right)=\bar{a}\left(u_{0}, u_{0}\right),
$$


since $\bar{u}_{0}$ satisfies the system of equations (5.12)-(5.17). Finally, by the symmetry of $a^{\epsilon}(\cdot, \cdot)$, we have

$$
\lim _{\epsilon \rightarrow 0} a^{\epsilon}\left(u^{\epsilon}-u_{0}^{\epsilon}, u^{\epsilon}-u_{0}^{\epsilon}\right)=0
$$

and by Lemma 3.2, we see that $\lim _{\epsilon \rightarrow 0}\left\{\left\|u^{\epsilon}-u_{0}^{\epsilon}\right\|_{L^{2}\left(\Omega^{\epsilon}\right)}+\epsilon\left\|\nabla\left(u_{s}^{\epsilon}-u_{0, s}^{\epsilon}\right)\right\|_{L^{2}\left(\Omega_{s}\right)}\right\}=0$.

Now we show the strong convergence of the pressure $p^{\epsilon}$ to $p_{0}$ in $W$. By the inf-sup condition for Stokes,

$$
\sup _{v \in H_{0}^{1}} \frac{(w, \nabla \cdot v)}{\|v\|_{1}}>\gamma\|w\|_{0}
$$

there exists a sequence $v^{\epsilon}$ such that $\left\|v^{\epsilon}\right\|_{1}=1$ and

$$
\left(p^{\epsilon}-p_{0}, \nabla \cdot v^{\epsilon}\right)_{\Omega^{\epsilon}}>\frac{\gamma}{2}\left\|p^{\epsilon}-p_{0}\right\|_{0}
$$

By Theorem 3.5 in Appendix A of [14], there exists $v_{0}(x) \in H^{1}(\Omega)$ and $v_{1}(x, y) \in$ $L^{2}\left(\Omega ; H_{\#}^{1}(Y) / \mathbb{R}\right)$ such that (possibly after passing to a subsequence)

$$
v^{\epsilon} \longrightarrow v_{0} \quad \text { and } \quad \nabla v^{\epsilon} \longrightarrow \nabla_{x} v_{0}(x)+\nabla_{y} v_{1}(x, y) .
$$

Note that we can assume $v_{1}$ is such that its average over $Y$ is zero. It follows that

$$
\lim _{\epsilon \rightarrow 0}\left(p_{0}, \nabla \cdot v^{\epsilon}\right)_{\Omega^{\epsilon}}=\left(p_{0}, \nabla \cdot v_{0}+\int_{Y} \nabla v_{1} d y\right)_{\Omega}=\left(p_{0}, \nabla \cdot v_{0}\right)_{\Omega},
$$

by the periodicity of $v_{1}$. Now,

$$
\begin{aligned}
\lim _{\epsilon \rightarrow 0}\left(p^{\epsilon}, \nabla \cdot v^{\epsilon}\right)_{\Omega^{\epsilon}} & =\lim _{\epsilon \rightarrow 0}\left(p^{\epsilon}, \nabla \cdot\left(v^{\epsilon}-v_{0}\right)\right)_{\Omega^{\epsilon}}+\left(p_{0}, \nabla \cdot v_{0}\right)_{\Omega} \\
& =\lim _{\epsilon \rightarrow 0} a^{\epsilon}\left(u^{\epsilon}, v^{\epsilon}-v_{0}\right)+\left(p_{0}, \nabla \cdot v_{0}\right)_{\Omega} .
\end{aligned}
$$

Finally, we can show

$$
\begin{aligned}
\lim _{\epsilon \rightarrow 0} a^{\epsilon} & \left(u^{\epsilon}, v^{\epsilon}-v_{0}\right) \\
= & \lim _{\epsilon \rightarrow 0}\left\{2 \mu\left(\epsilon D u^{\epsilon}, \epsilon D\left(v^{\epsilon}-v_{0}\right)\right)_{\Omega_{s}^{\epsilon}}\right. \\
& \left.+\left(\mu \alpha \sqrt{\frac{\epsilon}{K}} u_{s}^{\epsilon} \cdot \tau, \sqrt{\epsilon}\left(v^{\epsilon}-v_{0}\right)_{s} \cdot \tau\right)_{\Gamma^{\epsilon}}+\left(\mu K^{-1} u^{\epsilon}, v^{\epsilon}-v_{0}\right)_{\Omega_{d}^{\epsilon}}\right\} \\
= & \lim _{\epsilon \rightarrow 0}\left\{2 \mu\left(\epsilon D\left(u^{\epsilon}-u_{0}^{\epsilon}\right), \epsilon D\left(v^{\epsilon}-v_{0}\right)\right)_{\Omega_{s}^{\epsilon}}\right. \\
& +\left(\mu \alpha \sqrt{\frac{\epsilon}{K}}\left(u^{\epsilon}-u_{0}^{\epsilon}\right)_{s} \cdot \tau, \sqrt{\epsilon}\left(v^{\epsilon}-v_{0}\right)_{s} \cdot \tau\right)_{\Gamma^{\epsilon}} \\
& +\left(\mu K^{-1}\left(u^{\epsilon}-u_{0}\right), v^{\epsilon}-v_{0}\right)_{\Omega_{d}^{\epsilon}} \\
& +2 \mu\left(\epsilon D u_{0}^{\epsilon}, \epsilon D\left(v^{\epsilon}-v_{0}\right)\right)_{\Omega_{s}^{\epsilon}} \\
& +\left(\mu \alpha \sqrt{\frac{\epsilon}{K}} u_{0, s}^{\epsilon} \cdot \tau, \sqrt{\epsilon}\left(v^{\epsilon}-v_{0}\right)_{s} \cdot \tau\right)_{\Gamma^{\epsilon}} \\
& \left.+\left(\mu K^{-1} u_{0}^{\epsilon}, v^{\epsilon}-v_{0}\right)_{\Omega_{d}^{\epsilon}}\right\} \\
= & 0
\end{aligned}
$$


where the first three terms above converge to zero because $\lim _{\epsilon \rightarrow 0}\left\|u^{\epsilon}-u_{0}\right\|_{0}=0$, $\lim _{\epsilon \rightarrow 0} \epsilon\left\|\nabla\left(u^{\epsilon}-u_{0}^{\epsilon}\right)\right\|_{0}=0$, and because $v^{\epsilon}-v_{0}$ is bounded in $H^{1}$. The final three terms above converge to zero by the two-scale convergence results for $v^{\epsilon}$ (and because one term has an extra factor of $\epsilon$ ). Putting everything together, we have that

$$
\lim _{\epsilon \rightarrow 0}\left(p^{\epsilon}-p_{0}, \nabla \cdot v^{\epsilon}\right)=0
$$

as $\epsilon \rightarrow 0$. By (5.24), $p^{\epsilon} \rightarrow p_{0}$ as $\epsilon \rightarrow 0$ strongly in $W$.

6. A Simple Analytical Solution of the Auxiliary Problem. It is not so easy to construct analytical solutions to the auxiliary problem $(2.4)-(2.10)$, except at least in the following case. Let $Y=(0, \ell) \times(0, \ell)$ be a square of side length $\ell>0$. With $Y_{s}=(0, \ell) \times(0, h)$ and $Y_{d}=(0, \ell) \times(h, \ell)$ repeated periodically, we have a horizontally layered medium. Note that $\Gamma$ consists of two segments, $y_{2}=h$ and, by periodicity, $y_{2}=0 \Longleftrightarrow y_{2}=\ell$.

When $j=1$, it is easy to verify that the solution is

$$
\begin{aligned}
& \omega_{1}^{s}(y)=\frac{1}{2}\left(-y_{2}^{2}+h y_{2}+\frac{\sqrt{K}}{\alpha} h\right) e_{1}, \\
& \omega_{1}^{d}(y)=K e_{1}, \\
& \Phi_{1}(y)=0,
\end{aligned}
$$

which has flow in the $y_{1}$-direction only. It follows from (2.13) that $\tilde{K}_{21}=0$ (so $\tilde{K}$ is diagonal), and

$$
\tilde{K}_{11}=\frac{1}{\ell}\left(\frac{1}{12} h^{3}+\frac{\sqrt{K}}{2 \alpha} h^{2}+K(\ell-h)\right) .
$$

This should be contrasted to the situation in which the porous matrix is replaced by an impermeable medium. Then $\omega_{j}^{s}=0$ on $\Gamma$, and we have the well-known problem of Poiseuille flow in a pipe. The solution is

$$
\begin{aligned}
& \check{\omega}_{1}^{s}(y)=-\frac{1}{2} y_{2}\left(h-y_{2}\right) e_{1}, \\
& \check{\Phi}_{1}(y)=0 .
\end{aligned}
$$

In this case, we would compute the effective permeability for a unit pressure drop in the $y_{1}$-direction (which corresponds to the forcing function $e_{1}$ ) as

$$
\tilde{K}_{11, \text { Poiseuille }}=\frac{h^{3}}{12 \ell},
$$

which is the first term on the right side of (6.4).

If instead we assume the vugular region is impermeable, the bulk flow would be reduced from the porous medium case by the geometric factor $(\ell-h) / \ell$ :

$$
\tilde{K}_{11, \text { Darcy }}=\frac{\ell-h}{\ell} K \text {. }
$$

This is the last term on the right side of (6.4). Thus, when considering flow in the direction of the vugular channel, we have the representation

$$
\tilde{K}_{11}=\tilde{K}_{11, \text { Poiseuille }}+\tilde{K}_{11, \text { Beavers-Joseph }}+\tilde{K}_{11, \text { Darcy }},
$$


where

$$
\tilde{K}_{11, \text { Beavers-Joseph }}=\frac{\sqrt{K}}{2 \alpha \ell} h^{2}
$$

represents the Beavers-Joseph interface effect of fluid slippage. This term increases the Darcy-Stokes flow from the arithmetic average of the pure Stokes "pipe flow" and the pure Darcy flow. Note that we generally have $K \ll h^{2}$ and $\alpha=O(1)$. Thus

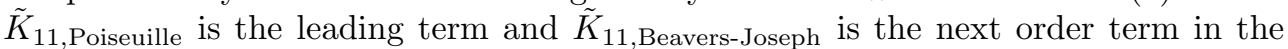
expansion.

When $j=2$ in the auxiliary problem $(2.4)-(2.10)$, it is easy to verify that the solution is

$$
\begin{aligned}
\omega_{2}(y) & =\frac{\ell}{\ell-h} K e_{2}, \\
\Phi_{2}^{s}(y) & =y_{2}, \\
\Phi_{2}^{d}(y) & =\frac{h}{\ell-h}\left(\ell-y_{2}\right) .
\end{aligned}
$$

Again $\tilde{K}_{12}=\tilde{K}_{21}=0$ and

$$
\tilde{K}_{22}=\frac{\ell}{\ell-h} K
$$

In this case, the flow is entirely in the $y_{2}$-direction.

It is well known and easily verified that one dimensional flow across a porous medium of permeability $k_{1}$ for distance $h$ and $k_{2}$ for distance $\ell-h$ results in a flow rate that is the same as that in a uniform medium of permeability $k=\frac{\ell k_{1} k_{2}}{h k_{2}+(\ell-h) k_{1}}$, which is the harmonic average permeability. If we apply this result to our case, assuming that the vugular channel has infinite permeability, we obtain exactly (6.14).

In conclusion, this example suggests that the effective permeability represents an average of two extremes. When the vugs are interconnected in some direction, we have primarily Poiseuille flow behavior, with a low order correction term for the effective permeability related to the Beavers-Joseph slip (and an even lower order correction related to flow entirely in the porous matrix). When the flow is along paths that alternate from vug to matrix, the fluid behaves as if it were flowing in a porous medium with the vugs having infinite permeability.

7. Acknowledgment. We thank the anonymous referee for suggesting Theorem 5.2, and the U.S. National Science Foundation for supporting this work (under grants DMS-0074310 and DMS-0417431).

\section{REFERENCES}

[1] G. Allaire, Homogenization and two-scale convergence, Siam J. Math. Anal., 23 (1992), pp. $1482-1518$.

[2] G. Allaire, A. Damlamain, and U. Hornung, Two-scale Convergence on Periodic Surfaces and Applications, Proceedings of the International Conference on Mathematical Modelling of Flow Through Porous Media (May 1995), A. Bourgeat et al., eds, pp. 15-25, World Scientific Pub., Singapore, 1996.

[3] T. Arbogast and D. S. Brunson, A computational method for approximating a Darcy-Stokes system governing a vuggy porous medium, to appear. 
[4] T. Arbogast, J. Douglas, JR., And U. Hornung, Derivation of the double porosity model of single phase flow via homogenization theory, SIAM J. Math. Anal., 21 (1990), pp. 823-836.

[5] I. BABUŠKA, The finite element method with Lagrangian multipliers, Numer. Math., 20 (1973), pp. 179-192.

[6] J. Bear, Dynamics of Fluids in Porous Media, Dover, New York, 1972.

[7] G. S. Beavers and D. D. Joseph, Boundary conditions at a naturally permeable wall, J. Fluid Mech., 30 (1967), pp. 197-207.

[8] A. Bensoussan, J. L. Lions, and G. Papanicolaou, Asymptotic Analysis for Periodic Structure, North Holland, Amsterdam, 1978.

[9] G. Bouchitté and I. Fragala, Homogenization of Thin Stuctures by the Two-Scale Method With Respect to Measures, SIAM J. Math. Anal., 32 (2001), no. 6, 1198-1226.

[10] S. C. Brenner And L. R. Scott, The Mathematical Theory of Finite Element Methods, Springer-Verlag, New York, 1994.

[11] F. BREzzI, On the existence, uniqueness and approximation of saddle-point problems arising from Lagrangian multipliers, RAIRO, 8 (1974), pp. 129-151.

[12] F. Brezzi And M. Fortin, Mixed and hybrid finite element methods, Springer-Verlag, New York, 1991.

[13] D. K. Garthing, C. E. Hickox, And R. C. Givler, Simulation of coupled viscous and porous flow problems, Comp. Fluid Dyn., 7 (1996), pp. 23-48.

[14] U. Hornung, ed., Homogenization and Porous Media, Interdisciplinary Applied Mathematics Series, Springer-Verlag, New York, 1997.

[15] W. JÄGER AND A. Mikelić, On the boundary conditions at the contact interface between a porous medium and a free fluid, Annali della Scuola Normale Superiore di Pise, Classe Fisiche e Matematiche-Serie IV, 23 (1996), pp. 403-465.

[16] - On the interface boundary condition of Beavers, Joseph, and Saffman, SIAM J. Appl. Math., 60 (2000), pp. 1111-1127.

[17] V. V. Jikov, S. M. Kozlov, And O. A. Oleinik, Homogenization of Differential Operators and Integral Functions, Springer-Verlag, New York, 1994.

[18] I. P. Jones, Low Reynolds number flow past a porous spherical shell, Proc. Camb. Phil. Soc., 73 (1973), pp. 231-238.

[19] W. J. Layton, F. Schieweck, And I. Yotov, Coupling fluid flow with porous media flow, SIAM. J. Numer. Anal., 40 (2003), pp. 2195-2218.

[20] J. L. Lions and E. Magenes, Non-Homogeneous Boundary Value Problems and Applications 1, Springer-Verlag, Berlin, 1970.

[21] M. Neuss-Radu, Some extensions of two-scale convergence, C. R. Acad. Sci. Paris Sér. I Math., 322 (1996), pp. 899-904.

[22] G. NGuetseng, A general convergence result for a functional related to the theory of homogenization, SIAM J. Math. Anal., 20 (1989), pp. 608-623.

[23] D. W. Peaceman, Fundamentals of numerical reservoir simulation, Elsevier, Amsterdam, 1977.

[24] P. G. SAFFman, On the boundary condition at the interface of a porous medium, Studies in Applied Mathematics, 1 (1971), pp. 93-101.

[25] A. G. SAlinger, R. ARIS, AND J. J. DERBY, Finite element formulations for large-scale, coupled flows in adjacent porous and open fluid domains, International Journal for Numerical Methods in Fluids, 18 (1994), pp. 1185-1209.

[26] E. Sanchez-Palencia, Non-homogeneous Media and Vibration Theory, no. 127 in Lecture Notes in Physics, Springer-Verlag, New York, 1980.

[27] L. TARTAR, Incompressible fluid flow in a porous medium-convergence of the homogenization process, in Non-homogeneous Media and Vibration Theory, E. Sanchez-Palencia, Lecture Notes in Physics 127, Springer-Verlag, Berlin, 1980, pp. 368-377.

[28] R. Temam, Navier-Stokes equations, theory and numerical analysis, North-Holland, Amsterdam, 2 ed., 1979.

[29] S. Whitaker, Flow in porous media I: A theoretical derivation of Darcy's law, Transport in Porous Media, 1 (1986), pp. 3-25. 